IMPACTOS DO IMPAIRMENT TEST NAS VARIÁVEIS CONTÁBEIS E NOS INDICADORES DE DESEMPENHO DAS 50 MAIORES COMPANHIAS LISTADAS NA BM\&FBOVESPA

\title{
$\mathrm{R} C \& \mathrm{C}$
}

REVISTA DE CDNTABILIDADE E CDNTRDLADDRIA

\section{IMPACTOS DO IMPAIRMENT TEST NAS VARIÁVEIS CONTÁBEIS E NOS INDICADORES DE DESEMPENHO DAS 50 MAIORES COMPANHIAS LISTADAS NA BM\&FBOVESPA}

\section{IMPACTS OF THE IMPAIRMENT TEST IN THE ACCOUNTING VARIABLES AND PERFORMANCE INDICATORS OF THE 50 LARGEST COMPANIES LISTED ON BM\&FBOVESPA}

Recebido em 07.08.2014 | Aceite final em 05.06.2015 | Nota: este artigo foi aceito pelo Editor Jorge Eduardo Scarpin e passou por uma avaliação double blind review A reprodução dos artigos, total ou parcial, pode ser feita desde que citada a fonte.

DAVID VICTDR RDCHA DD NASCIMENTD
Mestrando em Ciências Contábeis pela Universidade de Brasília (UnB) | Campus
Universitário Darcy Ribeiro | Asa Norte | Brasília - DF | CEP 70.910-900| Telefone (61)
8244-7094 | E-mail: davidvictorrocha@gmail.com |

JDSIMAR PIRES DA SILVA Mestrando em Ciências Contábeis pela Universidade de Brasília (UnB) | Campus Universitário Darcy Ribeiro | Asa Norte | Brasília - DF | CEP 70.910-900| Telefone (61) 8244-7094 | E-mail: josimarnx@yahoo.com.br |

\section{TATIANE SILVA SÁ} Mestranda em Ciências Contábeis pela Universidade de Brasília (UnB) | Campus Universitário Darcy Ribeiro | Asa Norte | Brasília - DF | CEP 70.910-900| Telefone (61) 8244-7094 | E-mail: tatianesilva85@yahoo.com.br |

\section{TIAGD JDSÉ GDNZAGA BDRGES} Mestrando em Ciências Contábeis pela Universidade de Brasília (UnB) | Campus Universitário Darcy Ribeiro | Asa Norte | Brasília - DF | CEP 70.910-900| Telefone (61) 8244-7094 | E-mail: tiagoborges32@gmail.com |

\begin{abstract}
RESUMO
Este artigo objetiva evidenciar os impactos gerados pelo reconhecimento das perdas por redução ao valor recuperável de ativos, nas variáveis contábeis e indicadores de desempenho das 50 maiores companhias - por faturamento listadas na BM\&FBOVESPA. Foram analisadas as demonstrações financeiras de 2010 a 2012. Observou-se que apnas 19 empresas reconheceram perdas por redução ao valor recuperável em ao menos um dos três períodos analisados. Adicionalmente, foram verificadas as variações provocadas no Ativo Total, Patrimônio Líquido, Lucro Líquido e nos indicadores de desempenho -
\end{abstract}


NASCIMENTO, David Victor Rocha do; SILVA, Josimar Pires da; SÁ, Tatiane Silva; BORGES, Tiago José Gonzaga.

Endividamento Geral, Margem EBIT e Margem Líquida. Os maiores efeitos do Impairment test foram constatados sobre o Lucro Líquido e o Patrimônio Líquido. Ademais, considerando que $38 \%$ da amostra utilizou o referido teste, houve um indicativo de avanço na representação dos valores de venda dos ativos, trazendo, a princípio, uma melhora na qualidade informacional para os usuários. Todavia, a subjetividade envolvida no processo, em relação ao valor justo e ao valor em uso, pode ensejar práticas de gerenciamento de resultados.

Palavras-Chave: Impairment test. Informações Contábeis. Indicadores de Desempenho. Valor Recuperável. Gerenciamento de resultados.

\begin{abstract}
This article aims to highlight the impacts generated by the recognition of losses on impairment of assets, in the accounting variables and performance indicators of the 50 largest companies - revenue - listed on the BM \& FBOVESPA. The financial statements from 2010 to 2012 were analyzed. It was observed that only 19 companies recorded losses for impairment on at least one of the three periods analyzed. Additionally, the variations caused in Total Assets, Equity, Net Income and performance indicators - General Debt, EBIT Margin and Net Margin - were observed. The major impacts of the impairment test were found on net income and equity. Moreover, considering that $38 \%$ of the sample used the above test, there was an indication of progress in representing the values of asset sales. It has brought an improvement in information quality for users. However, the subjectivity involved in the process, in relation to fair value and value in use, can give rise to practices of earnings management.
\end{abstract}

Keywords: Impairment Test. Accounting Information. Performance Indicators. Recoverable Amount. Earnings Management.

\title{
1 INTRDDUÇÃ̃
}

O processo de convergência às normas contábeis internacionais promoveu expressivas alterações das politicas e práticas contábeis adotadas no Brasil quanto à mensuração, reconhecimento e evidenciação dos elementos das demonstrações contábeis.

Esse processo foi alavancado pela Lei 11.638, de 28 de dezembro de 2007, decorrente do Projeto de Lei no 3.741/00. Nesse intermédio, entre a apresentação do projeto de lei e a criação da lei supracitada, foi criado o Comitê de Pronunciamentos Contábeis - CPC como resultado da união e esforços da Associação Brasileira de Companhias Abertas (ABRASCA), Associação dos Analistas e Profissionais de Investimento do Mercado de Capitais (APIMEC NACIONAL), Bolsa de Valores, Mercadorias e Futuros de São Paulo (BM\&FBOVESPA S.A.), Conselho Federal de Contabilidade (CFC), Fundação Instituto de Pesquisas Contábeis, Atuariais e Financeiras (FIPECAFI) e Instituto dos Auditores Independentes do Brasil (IBRACON). 
IMPACTOS DO IMPAIRMENT TEST NAS VARIÁVEIS CONTÁBEIS E NOS INDICADORES DE DESEMPENHO DAS 50 MAIORES COMPANHIAS LISTADAS NA BM\&FBOVESPA

Após a aprovação da Lei no 11.638/07, o CPC emitiu 46 pronunciamentos contábeis, titulados CPCs, apoiados nas normas internacionais emitidas pelo International Accounting Standard Board (IASB). Essas normas originam-se de traduções das International Financial Reporting Standard (IFRS) e das International Accounting Standard (IAS), transpostas à singularidade e às características das empresas brasileiras.

O primeiro desses pronunciamentos emitidos foi CPC 01 - Redução ao Valor Recuperável de Ativos (posteriormente sofrendo leves modificações, CPC 01 (R1)) no qual tem a premissa básica de que nenhum ativo deve figurar nas demonstrações contábeis por valores superiores ao seu valor recuperável.

As premissas oriundas deste pronunciamento e dos demais conduzem certo grau de subjetividade, como resultado da mudança de uma contabilidade baseada no modelo Code law, aproximando-se do modelo Common law, onde o julgamento profissional deve ser exercido possibilitando maneiras diferentes para mensuração, reconhecimento e evidenciação de determinados elementos, de forma que produza maior qualidade da informação que se propõe a representar. Nesse contexto, os gestores e profissionais da área contábil (em conjunto com laudos técnicos de demais profissionais afins) devem avaliar se os ativos estão registrados por valores superiores ao valor contábil e caso estejam, devem reconhecer a perda por Impairment.

Alicerçado nessa conjectura, idealizou-se a contígua indagação de pesquisa: Qual a influência do Impairment test nas variáveis Ativo, Patrimônio Líquido e Lucro Líquido e nos indicadores Endividamento Geral, Margem EBIT e Margem Líquida, nas 50 maiores companhias brasileiras de capital aberto, por faturamento, listadas na BM\&FBOVESPA? Logo, objetiva-se com esta pesquisa, evidenciar os impactos gerados pelo reconhecimento das perdas por redução ao valor recuperável de ativos nas variáveis contábeis e indicadores de desempenho das 50 maiores companhias por faturamento, que possuem ações listadas na BM\&FBOVESPA.

Salienta-se a magnitude deste trabalho em consequência da subjetividade envolvida no processo de mensuração dos ativos relacionados ao Impairment test. Esse teste, quando usado indevidamente ou quando não utilizado onde deveria, provoca efeitos nas demonstrações contábeis, acarretando perda da qualidade da informação, sobretudo no que concerne a um dos aspectos da representação fidedigna neutralidade -, o que pode interferir nas decisões dos usuários.

\section{FUNDAMENTAÇÃO TEÓRICA}

\subsection{REDUÇÃD AD VALDR RECUPERÁVEL DE ATIVDS}

O reconhecimento de perda por redução ao valor recuperável de ativos tem sido uma questão global desde meados da década de 1990. O Impairment test nasceu nos Estados Unidos, porque a confiabilidade dos relatórios financeiros se deteriorou devido à desvalorização discricionária de ativos fixos e ao gerenciamento de resultados por parte dos administradores. Esta desvalorização discricionária de ativos fixos afetou o resultado, influenciando seriamente as decisões dos investidores. Consequentemente, o Impairment test foi introduzido para promover normas contábeis e processuais para coibir imprudências de contabilidade dos administradores (YAMAMOTO, 2008).

O Financial Accounting Standard Board (FASB) divulgou o Statement of Financial Accounting Standards (SFAS) n. 121, Accounting for the Impairment of Long-Lived Assets and for Long-Lived Assets to Be Disposed, nos Estados Unidos, em 1995. Em seguida, em 1998, o International Accounting Standards 
NASCIMENTO, David Victor Rocha do; SILVA, Josimar Pires da; SÁ, Tatiane Silva; BORGES, Tiago José Gonzaga.

Committee (IASC) também emitiu a International Accounting Standards (IAS) n. 36, Impairment of Assets (YAMAMOTO, 2008).

Com o advento do processo de convergência no Brasil, foi emitido o CPC 01 - Redução ao valor recuperável de ativos - aprovado pelo Comitê de Pronunciamentos Contábeis em 14/07/07, atualizado posteriormente em 06/08/2010 (CPC 01 - R1), tendo como objetivo básico verificar a possível redução ao valor recuperável dos ativos. O referido Pronunciamento estabelece que a entidade deva assegurar-se de que seus ativos estejam registrados contabilmente por valor que não exceda seus valores de recuperação. Assim, a mensuração e o reconhecimento de ativos estão intimamente relacionados à capacidade de geração de fluxos de caixa futuros.

Smith (1994) afirma que se o valor do ativo diminui, ele sofreu prejuízo econômico. Se o valor presente líquido dos fluxos de caixa restantes fica abaixo do seu valor de abandono (o valor que poderia ser obtido por meio da venda ou outra alienação), o ativo deve ser abandonado e um teste de impairment realizado. Se um ativo é eliminado por um valor menor que o seu valor contábil, uma perda deve ser reconhecida. Se o valor do ativo diminui, mas permanece maior do que o seu valor de abandono, o ativo deve ser mantido. Nesse caso, o impairment não deve ser realizado porque não há nenhuma alienação. Enquanto o valor de um ativo permanece acima de seu valor contábil, não é reconhecida qualquer perda. No entanto, se o valor do ativo em tais circunstâncias declina e passa a valer menos do que o valor contábil, a perda pode ser reconhecida, mesmo que não haja nenhuma alienação.

Dessa forma, quando as expectativas de retorno de ativos forem diminuídas substancialmente em decorrência de situações adversas, tais ativos não devem permanecer evidenciados no Balanço Patrimonial da companhia pelo seu custo de aquisição depreciado, uma vez que não existe mais a capacidade de geração de benefícios econômicos futuros esperados quando da sua aquisição (STICKNEY e WEIL, 2002).

Para Santos et al (2003), Magalhães et al (2010), ludícibus et al (2010), CPC 01 (R1) (2011) e IAS 36, o valor contábil de um ativo ou unidade geradora de caixa - UGC (montante pelo qual o ativo está reconhecido no balanço depois da dedução de toda a respectiva depreciação, amortização ou exaustão acumulada e ajuste para perdas) não deve exceder ao valor recuperável (maior montante entre o seu valor justo líquido de despesa de venda e o seu valor em uso; isto é, o valor presente de fluxos de caixa descontado), conforme demonstrado na Figura 1.

Figura 1: Comparativo Impairment test.

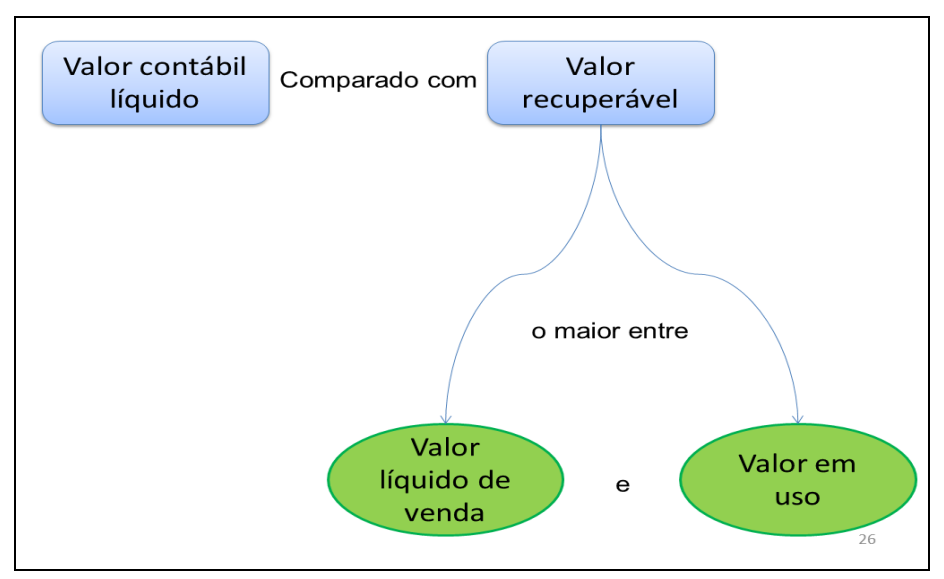

Fonte: Adaptado de Magalhães et al (2010).

Revista de Contabilidade e Controladoria, ISSN 1984-6266

Universidade Federal do Paraná, Curitiba, v. 7, n.2, p. 93-109, mai./ago. 2015. 
IMPACTOS DO IMPAIRMENT TEST NAS VARIÁVEIS CONTÁBEIS E NOS INDICADORES DE DESEMPENHO DAS 50 MAIORES COMPANHIAS LISTADAS NA BM\&FBOVESPA

Segundo Niyama et al (2010), o reconhecimento de uma desvalorização apurada em um ativo individual ou unidade geradora de caixa é efetuado mediante a constituição de uma provisão retificadora em contrapartida ao resultado do período ou à respectiva reserva de reavaliação. Dessa forma, a empresa reduz o ativo ao valor recuperável por meio da conta credora "perdas estimadas por redução ao valor recuperável", de forma similar à depreciação acumulada (IUDÍCIBUS ET AL, 2010).

\subsection{IMPAIRMENT TEST E ESTUDDS RELACIDNADDS ÀS NDRMAS INTERNACIDNAIS}

Diversas pesquisas foram realizadas no âmbito internacional, evidenciando o impacto da adoção das normas internacionais de contabilidade emitidas pelo IASB. Entre elas destacam-se aquelas relacionadas à qualidade da informação contábil e ao gerenciamento de resultados (ASHBAUGH e PINCUS, 2001; YOON, 2007; BARTH ET AL, 2008; CHRISTENSEN ET AL, 2007; PAANANEN e LIN, 2009; CHEN ET AL; 2009; CHEN ET AL, 2010; IATRIDIS, 2010;). Alguns estudos indicaram que a adoção das normas internacionais emitidas pelo IASB trouxe melhoria na qualidade da informação contábil (ASHBAUGH e PINCUS, 2001; YOON, 2007; BARTH ET AL, 2008; CHRISTENSEN ET AL, 2007; PAANANEN e LIN, 2009; CHEN ET AL, 2010; IATRIDIS, 2010). Uma possível explicação para esses resultados é a qualidade superior do padrão do IASB em relação ao conjunto de normas locais de diversos países, ao tratar de diversas situações não contempladas nas normas locais (KLANN e BEUREN, 2012). Outros evidenciaram que a adoção das IFRS resultou em aumento do gerenciamento de resultados (JEANJEAN e STOLOWY, 2008; ELBANNAN, 2011; KLANN e BEUREN, 2012). Segundo Klann e Beuren (2012), isso ocorre porque as normas contábeis baseadas em princípios (mais flexíveis) dariam mais liberdade de escolha para os gestores.

O estudo realizado por Ponte et al (2013) teve como objetivo principal a investigação das práticas de divulgação relativas ao teste de redução ao valor recuperável de ativos, no exercício de 2008 , adotadas pelas companhias abertas listadas na BM\&FBovespa. Para possibilitar melhor comparação das práticas adotadas, foram excluídas as empresas do setor financeiro e afins, devido às particularidades desse setor e às normas específicas emitidas pelo Banco Central para a categoria. A amostra foi composta por 337 empresas não financeiras, sendo 209 do mercado tradicional, 26 do Nível 1, 14 do Nível 2 e 88 do Novo Mercado. Os resultados evidenciaram que as empresas pesquisadas não adotaram integralmente as práticas de divulgação das informações do Impairment test, conforme as orientações do CPC 01. Todavia, destaca-se que mesmo não reconhecendo perdas por desvalorização dos ativos ou reversão de perdas, 199 empresas apresentaram, voluntariamente, informações sobre a realização do Impairment test, dado que nesta situação não estavam obrigadas.

O estudo realizado por Domingues (2009) teve como objetivo identificar o impacto das variações no preço do petróleo e no volume de reservas provadas sobre a perda do valor recuperável atribuída aos ativos de E\&P de empresas petrolíferas listadas na NYSE, durante o período que correspondeu aos relatórios contábeis de 2003 a 2008. Quanto à análise das normas que orientam o teste de Impairment, foi constatado que apesar das consideráveis semelhanças, as diferenças observadas podem distorcer o real objetivo do instrumento, que é o de garantir que os registros contábeis retratem o valor mais acurado possível dos benefícios econômicos futuros que os ativos podem gerar à entidade. No que tange à análise de como as variáveis selecionadas se relacionam e influenciam as perdas de Impairment de E\&P, observou-se a relação inversa dessa variável com os valores de Descobertas. 
NASCIMENTO, David Victor Rocha do; SILVA, Josimar Pires da; SÁ, Tatiane Silva; BORGES, Tiago José Gonzaga.

A pesquisa de Zhang et al (2010) examinou relatórios das despesas de imparidade de ativos das empresas chinesas, antes e após o anúncio de um novo padrão de contabilidade que proíbe a reversão das imparidades, dentro de um cenário relativamente único. Eles evidenciaram que as empresas listadas em bolsas de valores chinesas reconheceram menos imparidades durante o período de anúncio da transição, depois da nova norma e antes da data efetiva, do que no período pré-anúncio. Enquanto isso, as empresas com significativas baixas contábeis anteriores, revertem mais imparidades para alcançar as suas metas de lucros no período de transição.

\subsection{IMPAIRMENT TEST E GERENCIAMENTD DE RESULTADDS}

Segundo Laskaridou e Vazakidis (2013), o gerenciamento de resultados engloba transações com o objetivo de alcançar previsões de lucros preferenciais, aumento do preço das ações e a capacidade de gestão interna para otimizar o desempenho da empresa. Dessa forma, muitos estudos relacionados à suavização de resultados implicam que uma baixa contábil seja usada para neutralizar lucros líquidos anormais, a fim de criar reservas para os períodos posteriores.

Uma pesquisa realizada por Chen et al (2009) teve por objetivo examinar o gerenciamento de resultados através de reversões de Impairment de ativos induzida por incentivos regulatórios no mercado de ações da China. Com base em uma amostra de 2.370 casos de reversão ocorridos entre 2003 e 2006, os autores verificaram as seguintes constatações: 1 ) as empresas da amostra são motivadas por incentivos regulatórios para reverter prejuízos nos ativos para reduzir ou evitar a possibilidade de suspensão de negociação de suas ações; 2 ) foram verificadas evidências de que o value relevance da informação de inversão do impairment parece ser negativamente afetado pelo gerenciamento de resultados; 3 ) os mecanismos de monitoramento interno e externo desempenham um papel na limitação deste tipo de gerenciamento de resultados. Os autores concluíram que enquanto a intenção de realizar a reversão de impairment é um ato discricionário dos gestores a fim de revelar informações de recuperação de valor privado, os resultados encontrados no trabalho sugerem que um padrão contábil aparentemente melhor não leva necessariamente à divulgação nos relatórios financeiros.

Duh et al (2009) examinaram se a reversão de uma perda por imparidade anteriormente reconhecida fornece uma oportunidade para práticas de gerenciamento de resultados, e se tal comportamento está associado com os incentivos dos gestores. $O$ trabalho dos autores também examinou se os mecanismos de governança corporativa podem atenuar esse comportamento. A amostra do estudo é composta por 55 empresas que reverteram as perdas por imparidade entre 2005 e o primeiro trimestre de 2007. Os resultados mostraram que as empresas que reconhecem mais perdas por imparidade são mais propensas a reverter tais perdas por imparidade nos momentos em que isto evitaria uma queda dos lucros em um período subsequente. $\mathrm{O}$ artigo mostrou também que esse tipo de comportamento é mais acentuado em empresas que possuem uma proporção mais elevada de dívida, consistente com o gerenciamento de resultados ser associado com o incentivo para evitar a violação dos convênios da dívida. No entanto, um mecanismo eficaz de governança corporativa poderia mitigar esse tipo de comportamento.

Segundo Andrews (2012), o gerenciamento de resultados está relacionado com o teste de impairment devido à quantidade de escolhas discricionárias disponíveis pela gerência em uma decisão de realizar uma perda por imparidade e esse é um ponto importante porque os gestores podem utilizar a discricionariedade para manipular os resultados financeiros publicados. 
IMPACTOS DO IMPAIRMENT TEST NAS VARIÁVEIS CONTÁBEIS E NOS INDICADORES DE DESEMPENHO DAS 50 MAIORES COMPANHIAS LISTADAS NA BM\&FBOVESPA

Além disso, a manipulação de informações pode ser resultado de práticas de "big bath". Segundo Healy e Wahlen (1999) apud Andrews (2012), o termo "big bath" refere-se à prática de realizar uma grande baixa para "limpar o terreno". Para Laskaridou e Vazakidis (2013), a técnica do "big bath" é vista como um sinal para os investidores de que no balanço foi feito uma "limpa", ou seja, o balanço foi apresentado sem os recursos negativos que implicam em diminuir os retornos futuros.

O trabalho de Andrews (2012) investigou a prática de impairment de ativos em grandes corporações britânicas listadas antes e depois da mudança no ambiente regulatório para as International Financial Reporting Standard (IFRS). Os principais objetivos do trabalho foram verificar se uma perda por impairment no ativo resulta numa prevalência de gerenciamento de resultados ou em práticas de big bath accounting e verificar se mudanças no ambiente regulatório relativas ao teste de recuperabilidade de ativos resulta em um aumento de gerenciamento de resultados ou em práticas de big bath accounting. Os resultados do estudo apontaram que quando se consideram os encargos de depreciação de ativos ao longo de toda a amostra para o período de 2003 a 2007, isso indica que a característica predominante de gerenciamento de resultados é a suavização dos resultados ao invés da prática de big bath accounting. No entanto, após a mudança regulatória em 2005, houve um aumento da referida prática, quando comparado com o período anterior à mudança regulatória.

Laskaridou e Vazakidis (2013) fizeram um estudo que tinha como objetivo: trazer a questão da manipulação de impairment de ativos no contexto do gerenciamento dos resultados e examinar qualquer inferência estatística validando a discricionariedade do impairment e a experiência de ganhos das empresas. A amostra foi constituída de 26 empresas de alimentos e bebidas, listadas na Bolsa de Valores da Grécia, com base em observações de realização de impairment. Os níveis de rendimentos foram avaliados para dois grupos de empresas, as que realizaram o impairment e as que não realizaram, nos anos de 2005 e 2006. Os resultados demonstraram que as empresas que realizaram o impairment tiveram rendimentos inferiores aos seus homólogos que não possuem registros de baixas e que as perdas por imparidade são provavelmente relatadas como momento oportuno para as práticas de "big bath" e para aumentar os lucros futuros. No entanto, estima-se que o desempenho operacional das empresas gregas está relacionado com os ajustes dos resultados em ambiente tributável e com novas normas de contabilidade, do que com suscetíveis estratégias de rendimentos.

\section{METODOLOGIA}

\subsection{CRITÉRIDS DE PESQUISA}

Esta pesquisa utiliza-se dos seguintes critérios: exploratória, descritiva, bibliográfica, documental e quantitativa. No que tange ao procedimento documental, foram analisadas as demonstrações contábeis - Balanço Patrimonial, Demonstração de Resultado do Exercício e Notas Explicativas - das 50 maiores empresas por faturamento, listadas na BM\&FBOVESPA.

\subsection{DEFINIÇÃD DA AMDSTRA}

A amostra foi selecionada entre as companhias listadas na BM\&FBOVESPA que publicaram demonstrativos contábeis no período de 2010 a 2012. A partir desse grupo de empresas, foram extraídas aquelas que possuíam maior faturamento, conforme anuário da Revista Exame Melhores e Maiores. A seleção se deu analisando o ranking apresentado pela revista supracitada. Na ocorrência de empresas que figuravam no ranking, mas não são listadas na BM\&FBOVESPA, dirigia-se para a empresa 
NASCIMENTO, David Victor Rocha do; SILVA, Josimar Pires da; SÁ, Tatiane Silva; BORGES, Tiago José Gonzaga.

subsequente, de modo que foram consideradas apenas as empresas incluídas no ranking e que estavam listadas na BM\&FBOVESPA.

Foram selecionadas as 50 maiores empresas, por faturamento, e analisadas as Demonstrações de Resultado do Exercício, bem como as Notas Explicativas referentes aos períodos 2010, 2011 e 2012 de cada uma, com a intenção de investigar a ocorrência do reconhecimento de perda por redução ao valor recuperável em pelo menos um dos períodos.

As demonstrações contábeis (Balanço Patrimonial, Demonstração de Resultado e Notas Explicativas) coletadas para este estudo referem-se às demonstrações financeiras enviadas à BM\&FBOVESPA, via Sistema de Divulgação Externa (EmpresasNet).

Constatou-se que 19 das 50 empresas da amostra reconheceram perda por redução ao valor recuperável em ao menos um dos três períodos e que tais empresas pertenciam a sete setores: Petróleo, Materiais básicos, Consumo não cíclico, Construção e transporte, Telecomunicações, Utilidade pública e Consumo cíclico, conforme Figura 2.

Figura 2 - Distribuição das companhias por setor de atuação.

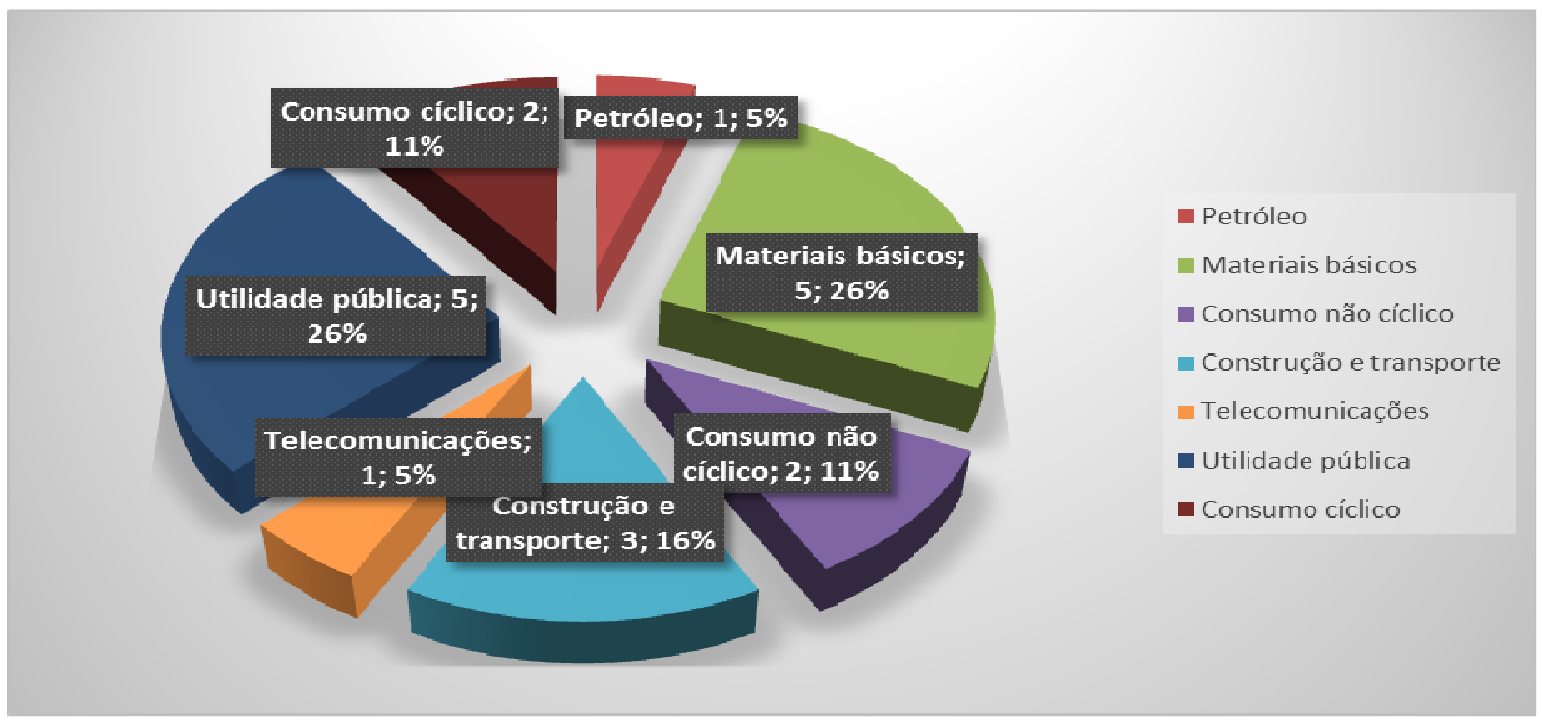

Fonte: elaborado pelos autores.

A partir da obtenção dessa amostra foram realizados ajustes no Balanço Patrimonial e Demonstração do Resultado do Exercício, com o intuito de obter demonstrações que não possuíssem o efeito do reconhecimento da perda por Impairment.

Foram coletadas as informações contábeis do Ativo Total, Patrimônio Líquido e Lucro Líquido e calculouse ainda os seguintes indicadores de desempenho: Endividamento Geral, Margem EBIT e Margem Líquida, com base nas demonstrações contábeis divulgadas. Ajustou-se os valores nas demonstrações contábeis com o objetivo de retirar o efeito da perda por Impairment, compará-las e avaliar as variações. 
IMPACTOS DO IMPAIRMENT TEST NAS VARIÁVEIS CONTÁBEIS E NOS INDICADORES DE DESEMPENHO DAS 50 MAIORES COMPANHIAS LISTADAS NA BM\&FBOVESPA

\section{ANÁLISE DOS RESULTADOS}

Nesta seção são veiculados os achados da pesquisa consoante à evidenciação dos impactos gerados pelo reconhecimento das perdas por redução ao valor recuperável de ativos nas variáveis contábeis e indicadores de desempenho das 50 maiores companhias por faturamento, que possuem ações listadas na BM\&FBOVESPA. Os dados obtidos foram reorganizados em quadros referentes aos três períodos analisados. O termo "NA (Não se aplica)" refere-se às empresas que não reconheceram perda ou reversão no referido período.

Pela análise do Quadro 1, nota-se que o reconhecimento de perdas por redução ao valor recuperável afetou consideravelmente $o$ ativo de parte das empresas analisadas. $O$ destaque ocorreu na variação do ativo total das companhias Magazine Luiza, Vale S.A., Celesc e Lojas Renner, onde, destas, a maior variação ocorreu na companhia Magazine Luiza, visto que nos três períodos que foi registrada a perda por Impairment ocorreram variações significativas e o Ativo Total seria superior em 4,46\%, 5,02\% e 5,98\%, respectivamente em 2010, 2011 e 2012, caso nenhuma perda por Impairment tivesse sido reconhecida.

A companhia Vale S.A. apresentou variação de 4,58\% em 2012 (único período de registro da perda), seguida da companhia Celesc, 3,76\% (2012) e Lojas Renner com 3,59\% (2010), 3,67\% (2011) e 3,34\% (2012). Acentuam-se, ainda, as variações de cerca de 1\% a 1,5\%, ocorridas nas empresas Gol, Eletrobrás e MRS Logística. As demais entidades apresentaram variações menores que $1 \%$ em todos os períodos.

Quadro 1: Efeito do impairment test no ativo total.

\begin{tabular}{|l|l|l|l|l|}
\hline \multirow{2}{*}{ Empresa } & \multicolumn{3}{l}{$\%$ variação } \\
\cline { 2 - 5 } & $\mathbf{2 0 1 0}$ & $\mathbf{2 0 1 1}$ & $\mathbf{2 0 1 2}$ \\
\hline $\mathbf{1}$ & PETROBRAS S.A. & $0,01 \%$ & $0,22 \%$ & $0,09 \%$ \\
\hline $\mathbf{2}$ & VALE S.A. & NA & NA & $\mathbf{4 , 5 8 \%}$ \\
\hline $\mathbf{3}$ & BRASKEM S.A. & NA & $-0,01 \%$ & $0,00 \%$ \\
\hline $\mathbf{4}$ & AMBEV & $-0,20 \%$ & NA & NA \\
\hline $\mathbf{5}$ & TAM & NA & $0,27 \%$ & $0,28 \%$ \\
\hline $\mathbf{6}$ & USIMINAS & $0,02 \%$ & $0,03 \%$ & $0,00 \%$ \\
\hline $\mathbf{7}$ & EMBRATEL & $0,03 \%$ & $0,11 \%$ & $0,10 \%$ \\
\hline $\mathbf{8}$ & GOL & NA & $0,47 \%$ & $1,33 \%$ \\
\hline $\mathbf{9}$ & ELETROBRAS & $0,09 \%$ & $0,32 \%$ & $1,44 \%$ \\
\hline $\mathbf{1 0}$ & COPEL & $0,25 \%$ & $0,10 \%$ & $0,17 \%$ \\
\hline $\mathbf{1 1}$ & MAGAZINE LUIZA & $\mathbf{4 , 4 6 \%}$ & $\mathbf{5 , 0 2} \%$ & $\mathbf{5 , 9 8 \%}$ \\
\hline $\mathbf{1 2}$ & COMGÁS & $0,13 \%$ & $0,48 \%$ & $0,50 \%$ \\
\hline $\mathbf{1 3}$ & CELESC & NA & NA & $3,76 \%$ \\
\hline $\mathbf{1 4}$ & FRIBRIA CELULOSE & $0,54 \%$ & $0,64 \%$ & $0,17 \%$ \\
\hline $\mathbf{1 5}$ & GERDAU & $-0,78 \%$ & NA & NA \\
\hline $\mathbf{1 6}$ & LOJAS RENNER & $\mathbf{3 , 5 9 \%}$ & $\mathbf{3 , 6 7 \%}$ & $\mathbf{3 , 3 4 \%}$ \\
\hline $\mathbf{1 7}$ & HYPERMARCAS & NA & $0,10 \%$ & $0,07 \%$ \\
\hline & & & & \\
\hline
\end{tabular}

Revista de Contabilidade e Controladoria, ISSN 1984-6266

Universidade Federal do Paraná, Curitiba, v. 7, n.2, p. 93-109, mai./ago. 2015. 


\begin{tabular}{|l|l|l|l|l|}
18 & CESP & $0,56 \%$ & NA & NA \\
\hline 19 & MRS LOGISTICA & $1,36 \%$ & NA & NA \\
\hline
\end{tabular}

Fonte: elaborado pelos autores.

No tocante ao Patrimônio Líquido, o Quadro 2 reporta os destaques evidenciados quanto a esse quesito. A companhia Magazine Luiza registrou aumento de 369,62\% no ano base 2010, quando a perda por redução ao valor recuperável é retirada. O PL real que era 47.436 milhões passou para 222.769 milhões. Nos períodos seguintes, 2011 e 2012, apresentou variações de 39,44\% e 54,96\% respectivamente, podendo ser consideradas como variações substanciais para a companhia.

As companhias GOL, Celesc, Lojas Renner e Vale S.A. também exibiram variações apreciáveis e acima da média. O PL da primeira variou em 16,38\% em 2012; a segunda teve variação de 10,61\% no mesmo ano; a terceira teve variações significativas nos três períodos, sendo 8,63\% em 2010, 9,47\% em 2011 e 9,66\% em 2012 e a quarta (Vale S.A.) apresentou variação de 7,85\% em 2012. Isso significa afirmar que o PL dessas companhias seriam maiores caso nenhuma perda por redução ao valor recuperável tivesse sido reconhecida. Em outras companhias as variações permaneceram de 1\% a 3,7\%: Eletrobrás em 3,69\% (2012); MRS Logística em 3,09\% (2010); GOL em 2,29\% (2011); COMGÁS em 1,65\% (2011) e 1,32\% (2012); TAM em 1,41\% (2011) e 1,10\% (2012) e Fibria Celulose em 1,24\% (2011).

Quadro 2: Efeito do impairment test no patrimônio líquido.

\begin{tabular}{|c|c|c|c|c|}
\hline \multirow{2}{*}{\multicolumn{2}{|c|}{ Empresa }} & \multicolumn{3}{|c|}{ \% variação } \\
\hline & & \multirow{2}{*}{$\begin{array}{c}2010 \\
0,02 \%\end{array}$} & \multirow{2}{*}{$\begin{array}{c}2011 \\
0,40 \% \\
\end{array}$} & \multirow{2}{*}{$\begin{array}{c}2012 \\
0,18 \% \\
\end{array}$} \\
\hline 1 & PETROBRAS S.A. & & & \\
\hline 2 & VALE S.A. & NA & NA & $7,85 \%$ \\
\hline 3 & BRASKEM S.A. & NA & $-0,04 \%$ & $-0,02 \%$ \\
\hline 4 & AMBEV & $-0,34 \%$ & NA & NA \\
\hline 5 & TAM & NA & $1,41 \%$ & $1,10 \%$ \\
\hline 6 & USIMINAS & $0,04 \%$ & $0,05 \%$ & $0,00 \%$ \\
\hline 7 & EMBRATEL & $0,09 \%$ & $0,31 \%$ & $0,27 \%$ \\
\hline 8 & GOL & NA & $2,29 \%$ & $16,38 \%$ \\
\hline 9 & ELETROBRAS & $0,19 \%$ & $0,68 \%$ & $3,69 \%$ \\
\hline 10 & COPEL & $0,39 \%$ & $0,16 \%$ & $0,28 \%$ \\
\hline 11 & MAGAZINE LUIZA & $369,62 \%$ & $39,44 \%$ & $54,96 \%$ \\
\hline 12 & COMGÁS & $0,37 \%$ & $1,65 \%$ & $1,32 \%$ \\
\hline 13 & CELESC & NA & NA & $10,61 \%$ \\
\hline 14 & FRIBRIA CELULOSE & $1,06 \%$ & $1,24 \%$ & $0,32 \%$ \\
\hline 15 & GERDAU & $-1,67 \%$ & NA & NA \\
\hline 16 & LOJAS RENNER & $8,63 \%$ & $9,47 \%$ & $9,66 \%$ \\
\hline 17 & HYPERMARCAS & NA & $0,19 \%$ & $0,13 \%$ \\
\hline 18 & CESP & $0,95 \%$ & NA & NA \\
\hline 19 & MRS LOGISTICA & $3,09 \%$ & NA & NA \\
\hline
\end{tabular}

Fonte: elaborado pelos autores. 
IMPACTOS DO IMPAIRMENT TEST NAS VARIÁVEIS CONTÁBEIS E NOS INDICADORES DE DESEMPENHO DAS 50 MAIORES COMPANHIAS LISTADAS NA BM\&FBOVESPA

Ressalta-se ainda que a companhia Gerdau teria seu PL reduzido em 1,67\% em 2010, em caso de não reconhecimento da reversão da perda reconhecida. A perda correspondente a essa reversão foi reconhecida no resultado do ano de 2009 e em 2010 foi revertida parcialmente. As demais companhias tiveram variações inferiores a $1 \%$ em todos os três exercícios analisados neste estudo.

No que se refere ao lucro ou prejuízo líquido, variações expressivas foram constatadas na maioria das empresas analisadas e em praticamente todos os períodos, ocorrendo, além disso, enormes variações como é o caso da companhia Magazine Luiza, em que o ajuste, retirando a perda por redução ao valor recuperável da demonstração do resultado, provocou aumento percentual no resultado de $2.099,53 \%$ em 2011, devido ao aumento do lucro líquido de $R \$ 11.666$ milhões para 256.597 milhões e $5.018,81 \%$ em 2012, conforme incremento emanado do prejuízo de $R \$ 6.745$ milhões para lucro de $R \$ 331.774$ milhões.

O Quadro 3 apresenta o efeito ocorrido no lucro líquido das 19 companhias analisadas. Adicionalmente, a companhia TAM em 2012, Magazine Luiza em 2010, Vale S.A. em 2012 e Cesp em 2010, tiveram variações superiores a $100 \%$; isto é, apresentaram respectivamente $347,02 \%, 254,72 \%, 132,28 \%$ e $106,60 \%$.

Quadro 3: Efeito do impairment test no lucro líquido.

\begin{tabular}{|c|c|c|c|c|}
\hline \multirow{2}{*}{\multicolumn{2}{|c|}{ Empresa }} & \multicolumn{3}{|c|}{ \% variação } \\
\hline & & \multirow{2}{*}{$\begin{array}{c}2010 \\
0,21 \% \\
\end{array}$} & \multirow{2}{*}{$\begin{array}{c}2011 \\
4,00 \% \\
\end{array}$} & \multirow{2}{*}{$\begin{array}{c}2012 \\
3,02 \% \\
\end{array}$} \\
\hline 1 & PETROBRAS S.A. & & & \\
\hline 2 & VALE S.A. & NA & NA & $132,28 \%$ \\
\hline 3 & BRASKEM S.A. & NA & $-0,73 \%$ & $-0,15 \%$ \\
\hline 4 & AMBEV & $-1,10 \%$ & NA & NA \\
\hline 5 & TAM & NA & $7,05 \%$ & $347,02 \%$ \\
\hline 6 & USIMINAS & $0,51 \%$ & $1,72 \%$ & $0,07 \%$ \\
\hline 7 & EMBRATEL & $0,80 \%$ & $10,73 \%$ & $2,67 \%$ \\
\hline 8 & GOL & NA & $6,73 \%$ & $7,93 \%$ \\
\hline 9 & ELETROBRAS & $5,29 \%$ & $14,00 \%$ & $35,83 \%$ \\
\hline 10 & COPEL & $4,41 \%$ & $1,60 \%$ & $4,82 \%$ \\
\hline 11 & MAGAZINE LUIZA & $254,72 \%$ & $2099,53 \%$ & $5018,81 \%$ \\
\hline 12 & COMGÁS & $0,88 \%$ & $8,71 \%$ & $8,12 \%$ \\
\hline 13 & CELESC & NA & NA & $78,05 \%$ \\
\hline 14 & FRIBRIA CELULOSE & $30,81 \%$ & $16,21 \%$ & $7,05 \%$ \\
\hline 15 & GERDAU & $-13,69 \%$ & NA & NA \\
\hline 16 & LOJAS RENNER & $28,62 \%$ & $32,48 \%$ & $35,48 \%$ \\
\hline 17 & HYPERMARCAS & NA & $7,40 \%$ & $4,06 \%$ \\
\hline 18 & CESP & $106,60 \%$ & NA & NA \\
\hline 19 & MRS LOGISTICA & $14,13 \%$ & NA & NA \\
\hline
\end{tabular}

Fonte: elaborado pelos autores.

Conforme já abordado, a maioria das empresas apresentaram efeitos significativos no Lucro Líquido quando as perdas por redução ao valor recuperável são retiradas, inclusive cita-se a companhia 
NASCIMENTO, David Victor Rocha do; SILVA, Josimar Pires da; SÁ, Tatiane Silva; BORGES, Tiago José Gonzaga.

Hypermarcas, em 2011 e GOL em 2011 e 2012, em que ocasionou relevante efeito na redução de prejuízos.

O Quadro 4 apresenta o efeito da perda por Impairment no Endividamento Geral das 19 empresas analisadas. Caso nenhuma perda por redução ao valor recuperável tivesse sido reconhecida, mudanças significativas ocorreriam nas seguintes empresas: Magazine Luiza, Lojas Renner, Celesc, Vale S.A. e MRS Logística. Elas teriam redução nesse indicador, caso não reconhecessem perda por Impairment, o que resultaria em menor dependência de recursos de terceiros para financiar ativos.

Quadro 4: Efeito do impairment test no endividamento geral.

\begin{tabular}{|c|c|c|c|c|}
\hline \multirow{2}{*}{\multicolumn{2}{|c|}{ Empresa }} & \multicolumn{3}{|c|}{ \% variação } \\
\hline & & \multirow{2}{*}{$\begin{array}{c}2010 \\
-0,01 \% \\
\end{array}$} & \multirow{2}{*}{$\begin{array}{c}2011 \\
-0,10 \%\end{array}$} & \multirow{2}{*}{$\begin{array}{c}2012 \\
-0,05 \% \\
\end{array}$} \\
\hline 1 & PETROBRAS S.A. & & & \\
\hline 2 & VALE S.A. & NA & NA & $-1,82 \%$ \\
\hline 3 & BRASKEM S.A. & NA & $0,01 \%$ & $0,00 \%$ \\
\hline 4 & AMBEV & $0,08 \%$ & NA & NA \\
\hline 5 & TAM & NA & $-0,22 \%$ & $-0,21 \%$ \\
\hline 6 & USIMINAS & $-0,01 \%$ & $-0,01 \%$ & $0,00 \%$ \\
\hline 7 & EMBRATEL & $-0,02 \%$ & $-0,07 \%$ & $-0,06 \%$ \\
\hline 8 & GOL & NA & $-0,37 \%$ & $-1,21 \%$ \\
\hline 9 & ELETROBRAS & $-0,05 \%$ & $-0,17 \%$ & $-0,87 \%$ \\
\hline 10 & COPEL & $-0,09 \%$ & $-0,04 \%$ & $-0,07 \%$ \\
\hline 11 & MAGAZINE LUIZA & $-4,27 \%$ & $-4,78 \%$ & $-3,17 \%$ \\
\hline 12 & COMGÁS & $-0,09 \%$ & $-0,34 \%$ & $-0,31 \%$ \\
\hline 13 & CELESC & NA & NA & $-2,34 \%$ \\
\hline 14 & FRIBRIA CELULOSE & $-0,26 \%$ & $0,00 \%$ & $0,00 \%$ \\
\hline 15 & GERDAU & $0,00 \%$ & NA & NA \\
\hline 16 & LOJAS RENNER & $-3,47 \%$ & $-3,54 \%$ & $-2,12 \%$ \\
\hline 17 & HYPERMARCAS & NA & $-0,05 \%$ & $-0,03 \%$ \\
\hline 18 & CESP & $-0,55 \%$ & NA & NA \\
\hline 19 & MRS LOGISTICA & $-1,34 \%$ & NA & NA \\
\hline
\end{tabular}

Fonte: elaborado pelos autores.

A dependência de recursos de terceiros da companhia Magazine Luiza seria reduzida em 4,27\%, 4,78\% e 3,17\% nos anos 2010, 2011 e 2012 respectivamente. As Lojas Renner teriam uma redução de 3,47\%, $3,54 \%$ e $2,12 \%$ no mesmo período e as companhias Celesc, Vale S.A., GOL e MRS Logística teriam reduções respectivas nesse indicador em 2,34\%, 1,82\%, 1,21\%, (todas em 2012) e 1,34\% (em 2010). As demais empresas apresentaram oscilações inferiores a $1 \%$.

O Quadro 5 mostra o efeito sobre a Margem EBIT. O destaque ocorreu na companhia Vale S.A. em 2012, em que o ajuste na demonstração do resultado (retirada da perda por Impairment) resultou em elevação desse indicador em 13,06 pontos percentuais, isto é, a margem EBIT era $16,04 \%$, passando para $29,10 \%$. 
IMPACTOS DO IMPAIRMENT TEST NAS VARIÁVEIS CONTÁBEIS E NOS INDICADORES DE DESEMPENHO DAS 50 MAIORES COMPANHIAS LISTADAS NA BM\&FBOVESPA

Quadro 5: Efeito do impairment test na Margem EBIT.

\begin{tabular}{|c|c|c|c|c|}
\hline \multirow{2}{*}{\multicolumn{2}{|c|}{ Empresa }} & \multicolumn{3}{c|}{$\%$ variação } \\
\cline { 3 - 5 } $\mathbf{1}$ & PETROBRAS S.A. & $\mathbf{2 0 1 0}$ & $\mathbf{2 0 1 1}$ & $\mathbf{2 0 1 2}$ \\
\hline $\mathbf{2}$ & VALE S.A. & NA & NA & $\mathbf{1 3 , 0 6 \%}$ \\
\hline $\mathbf{3}$ & BRASKEM S.A. & NA & $-0,01 \%$ & $0,00 \%$ \\
\hline $\mathbf{4}$ & AMBEV & $-0,33 \%$ & NA & NA \\
\hline $\mathbf{5}$ & TAM & NA & $0,41 \%$ & $0,60 \%$ \\
\hline $\mathbf{6}$ & USIMINAS & $0,06 \%$ & $0,08 \%$ & $0,00 \%$ \\
\hline $\mathbf{7}$ & EMBRATEL & $0,05 \%$ & $0,19 \%$ & $0,15 \%$ \\
\hline $\mathbf{8}$ & GOL & NA & $0,67 \%$ & $1,48 \%$ \\
\hline $\mathbf{9}$ & ELETROBRAS & $0,45 \%$ & $1,55 \%$ & $\mathbf{6 , 2 8 \%}$ \\
\hline $\mathbf{1 0}$ & COPEL & $0,65 \%$ & $0,24 \%$ & $0,41 \%$ \\
\hline $\mathbf{1 1}$ & MAGAZINE LUIZA & $\mathbf{3 , 6 5 \%}$ & $\mathbf{3 , 8 2} \%$ & $\mathbf{4 , 4 2 \%}$ \\
\hline $\mathbf{1 2}$ & COMGÁS & $0,13 \%$ & $0,50 \%$ & $0,56 \%$ \\
\hline $\mathbf{1 3}$ & CELESC & NA & NA & $\mathbf{4 , 4 4 \%}$ \\
\hline $\mathbf{1 4}$ & FRIBRIA CELULOSE & $\mathbf{2 , 5 9 \%}$ & $\mathbf{3 , 0 7 \%}$ & $0,80 \%$ \\
\hline $\mathbf{1 5}$ & GERDAU & $-1,07 \%$ & NA & NA \\
\hline $\mathbf{1 6}$ & LOJAS RENNER & $\mathbf{3 , 2 0 \%}$ & $\mathbf{3 , 3 8 \%}$ & $\mathbf{3 , 2 6 \%}$ \\
\hline $\mathbf{1 7}$ & HYPERMARCAS & NA & $0,38 \%$ & $0,24 \%$ \\
\hline $\mathbf{1 8}$ & CESP & $\mathbf{3 , 4 1 \%}$ & NA & NA \\
\hline $\mathbf{1 9}$ & MRS LOGISTICA & $\mathbf{2 , 7 6 \%}$ & NA & NA \\
\hline
\end{tabular}

Fonte: elaborado pelos autores.

Ressalta-se a relevância alcançada na análise das companhias Eletrobrás (2012), Magazine Luiza (2010, 2011 e 2012), Celesc (2012), Fibria Celulose (2010 e 2011), Lojas Renner (2010, 2011 e 2012), Cesp (2010), MRS Logística (2010), que apresentaram variações relevantes nesse indicador.

Assim como na margem EBIT, o destaque em relação à Margem Líquida foi a companhia Vale S.A. no ano de 2012, onde ocorreu elevação do indicador em 13,06 pontos percentuais devido ao ajuste na demonstração do resultado (retirada da perda por Impairment), ou seja, a margem líquida era 9,87\%, passando para $22,93 \%$.

O Quadro 6 apresenta outras empresas nas quais se pode afirmar considerável relevância ocorrida na variação deste indicador. Estas companhias são: Eletrobrás em 2011 e 2012; Magazine Luiza em 2010, 2011 e 2012; Celesc em 2012; Fibria Celulose em 2010 e 2011; Lojas Renner em 2010, 2011 e 2012; Cesp em 2010; MRS Logística em 2010 e GOL em 2012. Ressalta-se a redução em 1,07 pontos percentuais na companhia Gerdau em 2010, devido ao ajuste (redução no resultado) da reversão parcial de perdas reconhecidas no exercício de 2009. 
NASCIMENTO, David Victor Rocha do; SILVA, Josimar Pires da; SÁ, Tatiane Silva; BORGES, Tiago José Gonzaga.

Quadro 6: Efeito do impairment test na Margem Líquida.

\begin{tabular}{|c|c|c|c|c|}
\hline \multirow{2}{*}{\multicolumn{2}{|c|}{ Empresa }} & \multicolumn{3}{c|}{$\%$ variação } \\
\cline { 3 - 5 } & PETROBRAS S.A. & $\mathbf{2 0 1 0}$ & $\mathbf{2 0 1 1}$ & $\mathbf{2 0 1 2}$ \\
\hline $\mathbf{2}$ & VALE S.A. & NA & NA & $\mathbf{1 3 , 0 6 \%}$ \\
\hline $\mathbf{3}$ & BRASKEM S.A. & NA & $-0,01 \%$ & $0,00 \%$ \\
\hline $\mathbf{4}$ & AMBEV & $-0,33 \%$ & NA & NA \\
\hline $\mathbf{5}$ & TAM & NA & $0,41 \%$ & $0,60 \%$ \\
\hline $\mathbf{6}$ & USIMINAS & $0,06 \%$ & $0,08 \%$ & $0,00 \%$ \\
\hline $\mathbf{7}$ & EMBRATEL & $0,05 \%$ & $0,19 \%$ & $0,15 \%$ \\
\hline $\mathbf{8}$ & GOL & NA & $0,67 \%$ & $1,48 \%$ \\
\hline $\mathbf{9}$ & ELETROBRAS & $0,45 \%$ & $1,55 \%$ & $\mathbf{6 , 2 8 \%}$ \\
\hline $\mathbf{1 0}$ & COPEL & $0,65 \%$ & $0,24 \%$ & $0,41 \%$ \\
\hline $\mathbf{1 1}$ & MAGAZINE LUIZA & $\mathbf{3 , 6 5 \%}$ & $\mathbf{3 , 8 2} \%$ & $\mathbf{4 , 4 2} \%$ \\
\hline $\mathbf{1 2}$ & COMGÁS & $0,13 \%$ & $0,50 \%$ & $0,56 \%$ \\
\hline $\mathbf{1 3}$ & CELESC & NA & NA & $\mathbf{4 , 4 4 \%}$ \\
\hline $\mathbf{1 4}$ & FRIBRIA CELULOSE & $\mathbf{2 , 5 9 \%}$ & $\mathbf{3 , 0 7 \%}$ & $0,80 \%$ \\
\hline $\mathbf{1 5}$ & GERDAU & $-1,07 \%$ & NA & NA \\
\hline $\mathbf{1 6}$ & LOJAS RENNER & $\mathbf{3 , 2 0 \%}$ & $\mathbf{3 , 3 8 \%}$ & $\mathbf{3 , 2 6 \%}$ \\
\hline $\mathbf{1 7}$ & HYPERMARCAS & NA & $0,38 \%$ & $0,24 \%$ \\
\hline $\mathbf{1 8}$ & CESP & $\mathbf{3 , 4 1 \%}$ & NA & NA \\
\hline $\mathbf{1 9}$ & MRS LOGISTICA & $\mathbf{2 , 7 6 \%}$ & NA & NA \\
\hline $\mathbf{F O n}$ & M & & \\
\hline
\end{tabular}

Fonte: elaborado pelos autores.

Cumpre salientar que o efeito da retirada da perda por impairment é o mesmo sobre os indicadores Margem EBIT e Margem Líquida, tendo em vista que a mesma perda é retirada sobre o EBIT (na Margem EBIT) e sobre o lucro líquido (na Margem Líquida). Dessa forma, apesar dos resultados dos indicadores serem diferentes, a variação anual para esses indicadores foi igual, gerando tabelas iguais.

\section{CONSIDERAÇÕES FINAIS}

A partir da análise dos relatórios financeiros - Balanço Patrimonial, Demonstração do Resultado e Notas Explicativas - buscou-se identificar as variações ocorridas nas variáveis Ativo Total, Patrimônio Líquido e Lucro Líquido e nos indicadores de desempenho - Endividamento Geral, Margem EBIT e Margem Líquida - das 19 companhias que registraram perdas (ou reversão) por redução ao valor recuperável de ativos, conforme determina o pronunciamento contábil (CPC 01 - R1).

Conforme indicaram as análises, a maioria das empresas investigadas tiveram variações expressivas em ao menos uma das variáveis ou dos indicadores de desempenho, sendo que, em algumas companhias essas oscilações relevantes ocorreram em todos os indicadores analisados, por consequência do reconhecimento das perdas por redução ao valor recuperável de ativos.

Revista de Contabilidade e Controladoria, ISSN 1984-6266

Universidade Federal do Paraná, Curitiba, v. 7, n.2, p. 93-109, mai./ago. 2015. 
IMPACTOS DO IMPAIRMENT TEST NAS VARIÁVEIS CONTÁBEIS E NOS INDICADORES DE DESEMPENHO DAS 50 MAIORES COMPANHIAS LISTADAS NA BM\&FBOVESPA

As maiores variações ocorreram no Lucro Líquido, no qual as variações foram relevantes na maioria das empresas analisadas, seguido pela margem EBIT e margem líquida. Em geral, as variações ocorridas entre os indicadores calculados com as demonstrações normais e os indicadores calculados após o ajuste que retirou o efeito do Impairment das mesmas demonstrações foram significativas para a maioria das empresas analisadas.

Essa variação, que ocorreu devido ao reconhecimento e reversão das perdas por redução ao valor recuperável de ativos, pode levar os gestores à realização de práticas de gerenciamento de resultado, como evidenciado por Chen, Wang \& Zhao (2009) em pesquisas realizadas na China, em que o reconhecimento e reversão no ano seguinte provocaram alterações significativas no resultado, evitando que a empresa incorresse em prejuízos em dois anos consecutivos.

Diante do exposto, o Impairment representa um avanço na contabilidade brasileira, possibilitando maior qualidade na informação contábil, de forma que os relatórios financeiros possam refletir a posição econômico-financeira verdadeira das empresas, haja vista que os ativos não deverão figurar no Balanço Patrimonial por valores superiores ao recuperável. Porém, devido à subjetividade envolvida no processo, tanto em relação ao valor justo como em relação ao valor em uso, o Impairment test pode ser usado para o gerenciamento de resultados.

Sob este prisma, estudos posteriores poderiam verificar se a hipótese de gerenciamento de resultados seria confirmada para a amostra adotada ou para outras empresas, no intuito de contrastar as perspectivas de melhoria da qualidade da informação - no que tange ao valor de venda dos ativos - com uma eventual redução na qualidade informacional, em função de práticas oportunistas, considerando que estas práticas poderiam induzir o processo de tomada de decisão dos usuários.

\section{REFERÊNCIAS}

ANDREWS, R. Fair Value, Earnings Management and Asset Impairment: The Impact of a Change in the Regulatory Environment. Procedia Economics and Finance, v. 2, p. 16-25, 2012.

ASHBAUGH, H.; PINCUS, M. Domestic accounting standards, international accounting standards and the predictability of earnings. Journal of Accounting Research, 39, 417-434, 2001.

BARTH, M. E.; LANDSMAN, W. R.; LANG, M. H. International Accounting Standards and accounting quality. Journal of Accounting Research, v. 46, n. 3, p. 467-498, Jun. 2008.

BM\&FBOVESPA. Empresas listadas. Disponível em: http:// <http://www.bmfbovespa.com.br/ciaslistadas/empresas-listadas/BuscaEmpresaListada.aspx?idioma=pt-br>Acesso em: 05.07.2014.

CHEN, H.; TANG, Q.; JIANG, Y.; LIN, Z. The role of accounting standards: evidence from the European Union. Journal of International Financial Management \& Accounting, v. 21, n. 3, p. 1-57, 2010.

CHEN, S.; WANG, Y.; ZHAO, Z. Regulatory incentives for earnings management through asset impairment reversals in China. Journal of Accounting, Auditing \& Finance, v. 24, n. 4, p. 589-620, 2009.

CHRISTENSEN, H. B.; LEE, E.; WALKER, M. Cross-sectional variation in the economic consequences of international accounting harmonization: the case of mandatory IFRS adoption in the UK. The International Journal of Accounting, v. 42, p. 341-379, 2007. 
NASCIMENTO, David Victor Rocha do; SILVA, Josimar Pires da; SÁ, Tatiane Silva; BORGES, Tiago José Gonzaga.

COMITÊ DE PRONUNCIAMENTOS CONTÁBEIS-CPC. Pronunciamento Técnico CPC 01 (R1) - Redução ao Valor Recuperável de Ativos. Disponível em: <http://www.cpc.org.br/CPC/DocumentosEmitidos/Pronunciamentos/Pronunciamento?ld=2> Acesso em: 01.07.2014.

DOMINGUES, J. C. A. Perda do valor de recuperação em ativos de exploração e produção de petróleo e gás, 2009. Dissertação (Mestrado em Controladoria e Contabilidade) - Programa de Pós-Graduação em Controladoria e Contabilidade do Departamento de Contabilidade da Faculdade de Economia, Administração e Contabilidade de Ribeirão Preto, Universidade de São Paulo, São Paulo.

DUH, R. R.; LEE, W. C.; LIN, C. C. Reversing an impairment loss and earnings management: The role of corporate governance. The International Journal of Accounting, v. 44, n. 2, p. 113-137, 2009.

ELBANNAN, M. A. Accounting and stock market effects of international accounting standards adoption in an emerging economy. Review of Quantitative Finance and Accounting. v. 36, n.2, p. 207-245, 2011.

EXAME MELHORES E MAIORES 2013. As 1000 maiores empresas do Brasil. Edição 1044E, Julho/2013. Edição Especial 40 anos.

IATRIDIS, G. International Financial Reporting Standards and the quality of financial statement information. International Review of Financial Analysis. v.19, n.3, p.193-204, 2010.

INTERNATIONAL ACCOUNTING STANDARD BOARD. IAS 36 - Impairment of Assets. Disponível em: < http://www.ifrs.org/Pages/default.aspx >. Acesso em 03.03.2014.

IUDíCIBUS, Sérgio de; MARTINS, Eliseu; GELBCKE, Ernesto R.; SANTOS, Ariovaldo dos. Manual de Contabilidade Societária. São Paulo: Atlas, 2010.

JEANJEAN, T.; STOLOWY, H. Do accounting standards matter? An exploratory analysis of earnings management before and after IFRS adoption. Journal of accounting and public policy, v. 27, n. 6, p. 480494, 2008.

KLANN, R. C.; BEUREN, I. M. Gerenciamento de Resultados: Análise comparativa de empresas brasileiras e inglesas antes e após a adoção das IRFS. In: EnANPAD, 2012, Rio de Janeiro. Anais... Rio de Janeiro: EnANPAD, 2012.

LASKARIDOU, E. C.; VAZAKIDIS, A. Detecting Asset Impairment Management: Some Evidence from Food and Beverage Listed Companies. Procedia Technology, v. 8, p. 493-497, 2013.

MAGALHÃES, F. A. S.; SANTOS, R. C.; COSTA, F. M. IAS-36 - Redução ao Valor Recuperável de Ativos. In: Ernst \& Young e FIPECAFI. Manual de normas internacionais de contabilidade: IFRS versus normas brasileiras. v. 2, p. 44-52. São Paulo, 2010.

NIYAMA, J. K.; RODRIGUES, J. M.; ONO, H. M. Disclosure sobre Impairment: uma análise comparativa das companhias abertas em 2008. Revista de contabilidade do mestrado em ciências contábeis da UERJ (online), Rio de Janeiro, v. 15, n.1, p. 67, jan/abril, 2010.

PAANANEN, M.; LIN, H. The development of accounting quality of IAS and IFRS over time: the case of Germany. Journal of International Accounting Research, v. 8, n. 1, 2009.

PONTE, V. M. R. P.; DE LUCA, M. M. M.; SOUSA, H. V.; CAVALCANTE, D. S. Práticas de Divulgação do Teste de Redução ao Valor Recuperável de Ativos Pelas Companhias Abertas Listadas na Bm\&Fbovespa. Revista Contabilidade Vista \& Revista, ISSN 0103-734X, Universidade Federal de Minas Gerais, Belo Horizonte, v. 22, n. 4, p.113-144, 2013. 
IMPACTOS DO IMPAIRMENT TEST NAS VARIÁVEIS CONTÁBEIS E NOS INDICADORES DE DESEMPENHO DAS 50 MAIORES COMPANHIAS LISTADAS NA BM\&FBOVESPA

SANTOS, J. L. S.; MACHADO, N. P.; SCHMIDT, P. Teste de Impairment para Ativos de Longa Duração: Tratamento Contábil de Acordo com o SFAS no 144. Contexto, Porto Alegre, v. 3, n. 5, 20 semestre 2003.

SMITH, K. J. Asset impairment disclosures: will accounting for asset impairment lead to performance impairment? Journal of Accountancy. 178.6, Dec. 1994.

STICKNEY, C. P.; WEIL, R. L. Financial accounting: an introduction to concepts, methods, and uses. 10th ed. Fort Worth: Dryden, 2002.

YAMAMOTO, T. Asset Impairment Accounting and Appraisers: Evidence from Japan. The Appraisal Journal, Spring 2008.

YOON, S. Accounting quality and international accounting convergence. 107 f., Tese (Doctor of Philosophy) - Faculty of the Graduate College of the Oklahoma State University. 2007.

ZHANG, R.; LU, Z.; YE, K. How do firms react to the prohibition of long-lived asset impairment reversals? Evidence from China. Journal of Accounting and Public Policy, v. 29, n. 5, p. 424-438, 2010. 